\title{
Henri de Régnier, tel qu'en lui-même, enfin?, sous la direction de Bertrand Vibert
}

\section{Ida Merello}

\section{Q OpenEdition}

1 Journals

\section{Edizione digitale}

URL: http://journals.openedition.org/studifrancesi/561

DOI: 10.4000/studifrancesi.561

ISSN: 2421-5856

\section{Editore}

Rosenberg \& Sellier

\section{Edizione cartacea}

Data di pubblicazione: 1 aprile 2015

Paginazione: 181-183

ISSN: 0039-2944

\section{Notizia bibliografica digitale}

Ida Merello, « Henri de Régnier, tel qu'en lui-même, enfin?, sous la direction de Bertrand Vibert », Studi Francesi [Online], 175 (LIX | I) | 2015, online dal 01 avril 2015, consultato il 18 septembre 2020. URL : http://journals.openedition.org/studifrancesi/561 ; DOI : https://doi.org/10.4000/studifrancesi.561

Questo documento è stato generato automaticamente il 18 settembre 2020.

\section{(c) $(1) \odot \odot$}

Studi Francesi è distribuita con Licenza Creative Commons Attribuzione - Non commerciale - Non opere derivate 4.0 Internazionale. 


\title{
Henri de Régnier, tel qu'en lui-même, enfin?, sous la direction de Bertrand Vibert
}

\author{
Ida Merello
}

\section{NOTIZIA}

Henri de Régnier, tel qu'en lui-même, enfin?, sous la direction de Bertrand VIBERT, Paris, Classiques Garnier 2014, pp. 333.

1 Nell'introduzione, B. VIBERT affronta la questione del valore dell'opera di Régnier, mostrando la necessità di svincolare il giudizio su un autore dalle sue caratteristiche innovative, e l'esigenza di riconoscere la «genialità» anche di un'opera discontinua e contro tendenza, rivolta sempre più al passato. R.A. RUIZ DE CASTILLA (pp. 25-36) si interroga sulla dipendenza di Régnier da Mallarmé: va considerato un discepolo, oppure un interlocutore che, pur subendo l'influenza dell'altro, ha dato il suo contributo proprio ai mardis di rue de Rome? L'A. appoggia la seconda ipotesi, mettendo in rilievo la qualità autoriale di Régnier.

2 Pierre LACHASSE (pp. 37-48) legge le prose di Régnier, a partire dagli inizi su «Lutèce», mettendo in evidenza la loro autonomia, legata a un gusto lirico e allegorico, non disgiunto dallo spirito beffardo di Villiers, e ben lontano dal realismo degli altri testi narrativi. L'A. coglie anche due elementi centrali della poetica: la rivelazione del fenomeno della memoria involontaria e l'indistinzione tra reale e immaginario, cui si accompagna la ricerca di una lingua allegorica. Evidenzia quindi il nesso tra prosa e poesia, accostandovi i Soirs intimes et mondains, opera sperimentale, dove $\mathrm{i}$ temi simbolici e allegorici tratteggiano uno spazio mentale, attraverso una lingua difficile.

Mireille Losco-LENA (pp. 49-62) prende in considerazione l'opera teatrale, in cui, come nelle poesie, gli elementi simbolici corrispondono a stati d'animo, inquietudini e angosce prossime al teatro di Maeterlinck, e si sofferma in particolare su La Gardienne, 
opera difficile da mettere in scena. L'A. ricorda come l'argomento simbolicamente rappresentato - il cammino verso la propria anima - fosse difficile da comprendere per lo spettatore medio, e come i registi pretendessero per questo il silenzio in sala. Sebbene l'A. riprenda il giudizio di Szondi, sul fatto che si tratti di una lezione di morale insipida, mette tuttavia in luce l'abilità dialettica della pièce.

Grazie all'osservazione attenta del mondo delle "petites revues», Julien schuH (pp. 63-80) mostra le modalità con cui si è verificata l'evoluzione di Régnier dagli esordi simbolisti a un nuovo classicismo, fatto per non dispiacere all'Accademia. In una prima fase, dagli inizi su «Lutèce», fino agli «Écrits pour l'art» di René Ghil, attraverso «La Décadence» e «Le Scapin», l'A. vede l'affermazione di una strategia di potere di Régnier all'ombra di Mallarmé. In seguito, dopo l'apertura alle riviste belghe della «Wallonie» e della "Jeune Belgique», Régnier opera una sempre più ampia occupazione degli spazi, con una piena affermazione di sé all'interno della politica di relazione delle «petites revues». L'A. rende conto quindi del progressivo slittamento di Régnier verso un ritorno all'ordine classico sempre più evidente, fino alla svolta del 1896, quando i simbolisti e i nouveaux poètes non lo riconoscono più come uno di loro, e gli attribuiscono delle mire di potere. In effetti J. Schuh conferma la svolta, che si manifesta in primis con la collaborazione alla «Revue des deux mondes» e si conferma nel 1912 con l'elezione all'Académie.

5 Françoise LERICHE (pp. 81-96) legge il primo romanzo di Régnier, Une double maîtresse, secondo un doppio punto di vista. Da una parte, vede nel racconto una riscrittura del Candide, dove a essere cacciata di casa è Cunégonde; dall'altra, sottraendo il testo a una lettura psicanalitica in chiave edipica che era già stata proposta, l'A. lo collega piuttosto a una concezione del rapporto con la donna derivato da Schopenhauer e da Hartmann. Le forme di abiezione e degrado che prende il desiderio sono viste come risposta antitetica allo slancio nietzschiano di Gide.

6 Carole fURMAneK (pp. 97-112) cerca invece gli elementi di ritorno al Parnasse presenti nelle due raccolte della Cité des eaux (1902) e La Sandale ailée (1906). La visione rivolta al passato di Versailles nella prima raccolta sembra voler suggerire anche un ritorno alla versificazione tradizionale; mentre Régnier mantiene delle libertà metriche. Allo stesso modo l'A. coglie la modulazione dei temi parnassiani e la trasformazione degli elementi descrittivi in paesaggi psichici. In tal modo, come già per molti poeti a lui contemporanei, il parnassianesimo sfuma in simbolismo senza una evidente soluzione di continuità.

7 Michel VIEGNES (pp. 113-128), soffermandosi in particolare su L'Entrevue, ha buon gioco nel sostenere il carattere poetico del fantastico di Régnier, dove appare evidente anche l'aspetto teatrale della quarta parete. L'Entrevue è fondata sulla declinazione dell'immagine dello specchio come universo parallelo, in cui conta non solo il funzionamento come doppio, ma anche la modalità di riflessione: lo specchio è infatti collegato all'elemento liquido nelle molteplici superfici riflettenti di tutti i vetri e le acque di Venezia. Il mondo parallelo emerge dalla fluidità, in un'intercambiabilità tra immagine e realtà che si manifesta anche nel rapporto tra il fantasma del passato e il protagonista vivente. L'A. sottolinea infine come la fêlure, la ferita alla testa che riporta il protagonista nel mondo dell'aldiquà, rappresenti anche una caratteristica delle anime fragili, ma che per questo accedono a una visione interiore.

8 Franck JAVOUREZ (pp. 129-142) analizza il rapporto tra humour, ironia e spirito libertino in Les rencontres de M. Bréot, pubblicate nel 1904, e Voyage d'amour, del 1930. Una lettura 
attenta delle sue opere permette all'A. di mostrare il gioco di Régnier con i registri, nell'ambito di un libertinismo leggero, dove il distacco ironico con esclude la dolcezza iniziale di impressione. In sostanza, conclude l'A., per Régnier il libertino è colui che ritrova le proprie origini animalesche.

Vladimir SCHотTER (pp. 143-166) prende invece in esame una plaquette di sedici sonetti, uscita nel 1927, con un metodo rigorosamente stilistico. In tal modo riesce a definire con esattezza l'influenza di Heredia, arrivando alla definizione di hérédèmes per gli stilemi da lui derivati. Tuttavia limita il suo modello a costellazioni di parole, usate soprattutto in rima, e a riprese tematiche, affermando invece che i sonetti nel loro insieme si offrono come la tessitura di un oggetto prezioso, e lasciano trasparire concezioni estetiche diverse, anche antitetiche.

10 Silvia ROVERA (pp. 185-198) riprende il concetto di luogo mentale dei paesaggi di Régnier, e gli attribuisce non solo il gusto per le case piene di ricordi, che sembrano consentire una sospensione del tempo, ma anche l'amore per la letteratura. Anche la leggenda di Psiche rappresenta un movimento interiore, che attraversa più raccolte di Régnier. L'incontro con Eros raffigura il fuoco della creazione, e viene ad assumere poi i tratti della memoria, e trasformarsi in libro, lettera, poesia.

11 Denis HERLIN (pp. 199-214) studia i rapporti tra Régnier e Débussy: l'uso del verso libero di Régnier ne aveva suggerito a Debussy la trasposizione musicale, e c'è chi intravede un legame tra i Nocturnes di Debussy e le Scènes au crépuscules di Régnier. Ma, come l'A. mette in evidenza, la prevalenza di versi regolari in Régnier e il ritorno definitivo all'ordine tengono lontano chi non considera la versificazione regolare adatta alla musica. Da parte sua invece Régnier dedica a Debussy un sonetto della raccolta Tel qu'un songe - di cui l'A. ha ritrovato la versione originale - e favorisce i suoi contatti con Maeterlinck per la riuscita di Pelléas e Mélisande. La rottura di Debussy con la prima moglie, che tentò il suicidio, fece invece calare il gelo tra i due artisti.

12 Bertrand VIBERT (pp. 215-232) prende l'immagine della flûte come filo conduttore dell'ispirazione di Régnier, a indicare sia il rapporto privilegiato con l'acqua, sia con Pan, legato al desiderio e all'istinto, sia con il respiro, della vita come della natura. In opposizione alla lira di Apollo, il flauto insomma riassorbe in sé tutta la qualità poetica. L'A. individua nella flûte anche il filo conduttore dei romanzi, dalle Rencontres de M. de Bréot (1904) fino a La Pécheresse (1920), mostrando inoltre come il personaggio del fauno percorra tutta l'opera narrativa, in un'evoluzione che mette in evidenza a partire dal 1900 la pacificazione ormai raggiunta tra carne e spirito.

Gilles PHILIPPE (pp. 233-245) inserisce le sue riflessioni nel dibattito sullo stile, e analizza la lingua di Régnier mostrando come, anche nel primo quarto del xx secolo, pur all'interno di un rinnovato classicismo, Régnier mantenga l'uso dell'animismo dei verbi pronominali legati ad oggetti, o il legame tra due astratti attraverso un verbo metaforico. Una lettura dei Cahiers consente all'A. di verificare l'evolversi dell'attenzione allo stile da parte di Régnier: sempre meno presente nel corso degli anni, tranne qualche caso sporadico. Pur divertendosi ai pastiches della lingua del XVIII secolo, Régnier non credette alla sua superiorità, mentre diede il sostanziale consenso agli stereotipi sullo stile degli autori della sua generazione, con un'attenzione maggiore nei confronti di Hugo, Michelet e France. Malgrado l'evoluzione in senso classico, Régnier resta convinto della necessità di innovazione della scrittura; mentre le sue prose sono sempre meno tese alla ricerca del bello stile, per una riflessione più ampia su quel che costituisce la qualità stilistica. 
Fanny DÉcHANET-PLATZ (pp. 247-260) esamina la persistenza dei luoghi e delle persone del passato in Régnier, persistenza che dà origine a fenomeni di spiritismo o di follia. Anche la memoria involontaria ha la sua parte, e l'A. ne coglie le analogie con Proust, ma anche le differenze, per l'innesco in Régnier di una rêverie fantastica e per il legame con il sogno. Proprio per queste differenze i rapporti tra Régnier e Proust non furono facili, ma l'A. ritrova in entrambi la matrice di Nerval. In conclusione, per l'A., Régnier scinde il passato dalla memoria, in quanto il passato si ripropone continuamente, senza esigere lo sforzo di ricordare.

Alain GUYOT (pp. 261-272) analizza le immagini veneziane di Régnier, che, pur rifiutando il lirismo, tende a far trascolorare il reale nell'immaginario e viceversa. Il richiamo al genere del racconto di viaggio è utile allo scrittore, nell'Altana, ad alternare lettere, frammenti di diario e racconti, tutti apparentati da questo rapporto tra fantasticheria e realtà.

Bernard вочкномоvsку (pp. 273-292) è attento al Régnier moralista, e attraverso un confronto serrato tra i Cahiers e Donc, raccolta di massime uscita nel 1927, pur dimostrando che Donc è integralmente contenuto nei Cahiers, con un quarto degli scritti del tutto identico e gli altri di poco modificati, ribadisce il valore della raccolta come opera à part entière, non tanto per la costruzione di serie tematiche, quanto per una modulazione dei materiali che rende necessaria la composizione, come in uno sviluppo musicale, che ruota intorno agli elementi centrali dell'amore, la solitudine, la mondanità, la malinconia, la noia, ecc. Anche gli aneddoti che vi compaiono costituiscono una suite fondata sul gusto delle singolarità bizzarre.

Patrick BESNIER (pp. 293-302) cita il lamento di Marcel Scwhob e di Émile Henriot sulla mancanza di scritti autobiografici di Régnier, per mostrare come in realtà i ricordi siano intessuti nei racconti e nei volumi di viaggio. L'A. rintraccia la prima pagina di Souvenirs nel n. 1 di «Entretiens politiques et littéraires», e vi individua già le caratteristiche presenti anche nelle opere successive: una scrittura oscura, per il timore di una parzialità di visione, la volontà di non esporsi mai in prima persona con commenti su personaggi pubblici e situazioni pubblicamente controverse. Per questo Régnier si rifugia in genericità impersonali, oppure nella storia familiare e nella genealogia, che gli è sempre stata a cuore. 